\title{
LETTER
}

\section{Delayed diagnosis of tooth aspiration in three multiple trauma patients with mechanical ventilation}

\author{
Mao Zhang', Guang-Ju Zhou*', Song Zhao², Jian-Xin Yang', Xiao Lu', Jian-Xin Gan' and Shao-Wen Xu'
}

Tooth aspiration is a critical and rare complication in trauma patients. It can remain undetected and misdiagnosed. Here we present three mechanically ventilated multiple trauma cases with delayed diagnosis of bronchial tooth aspiration.

\section{Case 1}

A 23-year-old man was severely injured in a car accident and had multiple trauma, with an injury severity score (ISS) of 50 and a head abbreviated injury score (AIS) of 5. Three days later he was transferred to our hospital and chest CT showed a tooth-like foreign body in the right bronchial tree, which was confirmed to be a bicuspid (4 according to the Palmer notation method) by flexible bronchoscopy. As the tooth was psilate and firmly embedded, many attempts with either flexible or rigid bronchoscopy could not extract it. The patient died of sepsis 70 days after the accident.

\section{Case 2}

A 26-year-old man was presented to a local hospital with multiple trauma (ISS 54, head AIS 5) following a motorcycle accident. On the next day when he was transferred to our hospital, a chest film and fibreoptic bronchoscopy confirmed a lateral incisor $(\lfloor 2)$ in the left lobe. We failed to extract the tooth, but fortunately it was expectorated via the tracheotomy tube following a vigorous cough on day 12 .

\section{Case 3}

A 25-year-old man was presented with multiple trauma (ISS 41, head AIS 4) after a motorcycle accident. He was transferred to our hospital 9 hours later. Chest CT and flexible bronchoscopy confirmed a central incisor $(1])$ in

\footnotetext{
*Correspondence: zhoutom1978@hotmail.com

'Department of Emergency Medicine, Second Affiliated Hospital, Zhejiang

University, School of Medicine, Research Institute of Emergency Medicine,

Zhejiang University, Hangzhou, 310009 China

Full list of author information is available at the end of the article
}

the right bronchial tree. It was extracted by flexible bronchoscopy on day 8 (Figure 1).

Early diagnosis of tooth aspiration in coma patients is difficult because aspiration is rarely considered in the absence of an acute clinical presentation. Thus, an endobronchial tooth can remain undetected for a long time and result in serious complications [1]. In all three cases reported here, tooth aspiration was missed by the local hospitals. Doctors should suspect that any tooth that has been avulsed and not found as possibly aspirated in trauma patients, especially for those in a coma status. The medical history, clinical signs and radiological findings should be carefully checked. CT is more accurate than chest radiography in detecting endobronchial foreign bodies. When negative chest radiography or doubtful clinical findings are presented, bronchoscopy should be performed to confirm the diagnosis [2]. Both flexible and rigid bronchoscopy can be used for the diagnosis and removal of teeth [3]. Tracheotomy may facilitate the removal of an aspirated tooth.

\section{Abbreviations \\ AIS, abbreviated injury score; CT, computed tomography; ISS, injury severity score.}

\section{Competing interests}

The authors declare that they have no competing interests.

\section{Acknowledgements}

Written consent for publication was obtained from the patients' next-of-kin.

\section{Author details}

'Department of Emergency Medicine, Second Affiliated Hospital, Zhejiang University, School of Medicine, Research Institute of Emergency Medicine, Zhejiang University, Hangzhou, 310009 China. ${ }^{2}$ Department of Radiology, Second Affiliated Hospital, Zhejiang University, School of Medicine, Hangzhou, 310009 China.

\section{Published: 12 May 2011}

\section{References}

1. Lee P, Culver DA, Farver C, Mehta AC: Syndrome of iron pill aspiration. Chest 2002, 121:1355-1357.

2. Yurdakul AS, Kanbay A, Kurul C, Yorgancilar D, Demircan S, Ekim N: An occult foreign body aspiration with bronchial anomaly mimicking asthma and pneumonia. Dent Traumatol 2007, 23:368-370.

3. Xiao WL, Zhang DZ, Wang YH: Aspiration of two permanent teeth during maxillofacial injuries. J Craniofac Surg 2009, 20:558-560. 

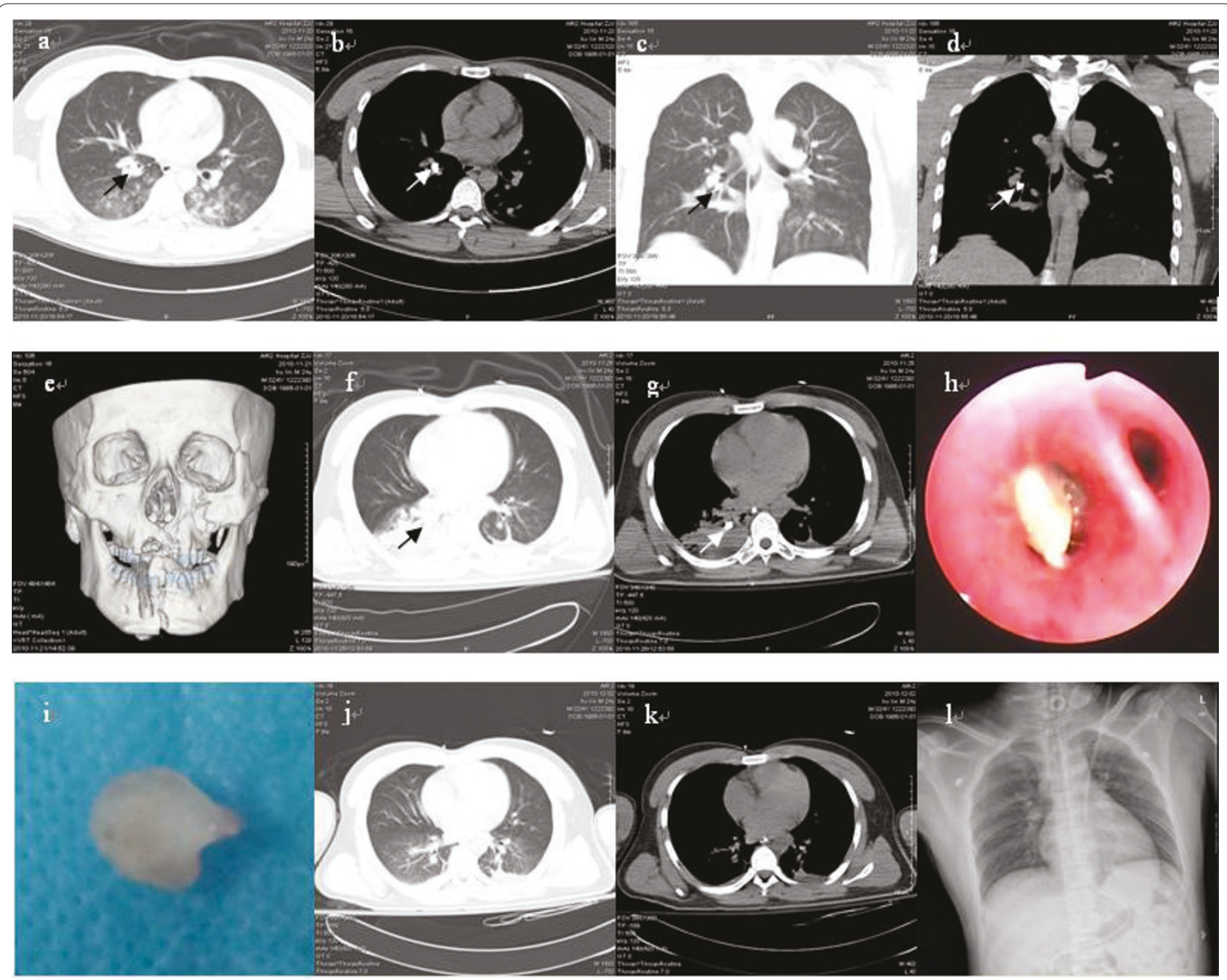

Figure 1. CT images of lung and maxillofacial bones, inspired tooth in case 3. (a-d) CT scan on day 1 showed the tooth-like foreign body (white/black arrow) in the right bronchus. (e) Reconstructed CT images on day 2 showed fracture of mandible and left zygomatic arch, and loss of three teeth. (f,g) Obstructive pneumonia developed in the right lower lobe on day 7. (h) Flexible bronchoscopy confirmed the tooth in the right bronchus. (i) The central incisor removed by flexible bronchoscopy on day 8. (j-I) Chest CT (day 13) and radiography (day 24) showed extinction of obstructive pneumonia in the right lower lobe.

doi:10.1186/cc10148

Cite this article as: Zhang M, et al:: Delayed diagnosis of tooth aspiration

in three multiple trauma patients with mechanical ventilation. Critical Care 2011, 15:424 